\title{
LES PETITES PROTÉINES G
}

\section{Jean de Gunzburg}

\section{RÉFÉRENCES}

1. Valencia A, Chardin P, Wittinghofer A, Sander C. The ras protein family : evolutionary tree and role of conserved amino acids. Biochemistry 1991; 30 : of conserve $4637-48$.

2. Chardin P. Small GTP-binding proteins of the ras family : a conserved functional mechanism? Cancer Cells $1991 ; 3: 117-26$.

3. Hall A. The cellular functions of small GTP binding proteins. Science $1990 ; 249: 635-40$

4. Wittinghofer A, Pai EF. The structure of Ras protein : a model for a universal molecular switch. Trends tein : a model for a univers
Biol Sci $1991 ; 16: 382-7$.

5. Rothman JE, Orci L. Molecular dissection of the secretory pathway. Nature $1992 ; 355$ : 409-15.

6. Bischoff FR, Ponstingl H. Mitotic regulator protein RCC1 is complexed with a nuclear ras-related polypeptide. Proc Natl Acad Sci USA 1991; 88: 10830-4.

7. Hancock JF, Magee AI, Childs JE, Marshall CJ. All ras proteins are polyisoprenylated but only some are palmitoylated. Cell $1989 ; 57: 1167-77$

8. de Gunzburg J. Protéine farnésyl et géranylgéranyl transférases. CR Soc Biol 1991; $185: 290-305$.

9. Chavrier P, Gorvel JP, Telzer E, Simons K, Gruenberg J, Zerial M. Hypervariable C-terminal domain acts as a targeting signal. Nature $1991 ; 353$. 769-72.

10. McCormick F. ras GTPase activating protein : signal transmitter and signal terminator. Cell 1989 ; 56 : $5-8$.

11. McCormick F. The world according to GAP. Oncogene 1990 ; 5 : 1281-3.

12. Filhol $\mathrm{O}$, Cochet $\mathrm{C}$. Le transfert des signaux mitogéniques: une affaire de particules. médecine/sciences $1990: 6: 980-4$

13. Diekman D, Brill S, Garrett MD, at al. Bat encodes a GTPase-activating protein for p2 $1^{\text {rac. Nature }}$ $1991 ; 351: 400-2$.

\section{ADRESSE}

J. de Gunzburg : chargé de recherche au Cnrs. Inserm U. 248, faculté de médecine Lariboisière - Saint-Louis, 10, avenue de Ver- es petites protéines $\mathrm{G}$ constituent une famille d'au moins une cinquantaine de protéines qui sont apparentées aux produits des protooncogènes ras et dont on retrouve des représentants dans les cellules de tous les organismes eucaryotes, des mammifères à la levure Saccharomyces cerevisiae, en passant par la drosophile et les nématodes [1-3]. Ces protéines, dont la masse moléculaire est généralement comprise entre 20000 et 30000 daltons, sont capables de lier le GDP et le GTP avec une forte affinité et possèdent une faible activité GTPase. Elles présentent entre elles d'importantes homologies de séquence qui traduisent une organisation très similaire de ces molécules en domaines structuraux et fonctionnels ; la structure tridimensionnelle de la protéine H-ras, prototype des protéines de cette famille, est connue depuis quelques années [4]. Selon des critères d'homologie de séquence, ces protéines ont été regroupées en trois principales sous-familles :

- la famille ras proprement dite, comprenant notamment les protéines H-ras, $\mathrm{K}$-ras et $\mathrm{N}$-ras qui sont probablement impliquées dans le contrôle de la prolifération cellulaire [3], ainsi que les protéines rap dont la fonction est encore inconnue ;

- la famille rho : les protéines rho A, B et C semblent jouer un rôle dans l'organisation du cytosquelette, notamment au niveau du réseau constitué par les câbles d'actine. Les protéines rac1 et rac2 participent directement au contrôle de l'activité de la NADPH oxydase des neutrophiles, enzyme responsable de la production d'ions superoxydes par ces cellules. La protéine $\mathrm{G} 25 \mathrm{~K} / \mathrm{CDC} 42 \mathrm{Hs}$, phosphorylée par le récepteur de l'EGF en réponse à la liaison de son ligand, pourrait être impliquée dans la réponse mitogène des cellules à ce facteur;

- la famille rab compte à présent une trentaine de membres, dont les protéines Ypt1 et Sec4 de la levure Saccharomyces cerevisiae, qui jouent un rôle important dans les processus de sécrétion chez cet organisme. Généralement localisées à la surface d'organites impliqués dans les voies d'endocytose ou de sécrétion, on pense que ces protéines interviennent dans la régulation du transport vésiculaire [5] (voir l'article de Bruno Goud, p. 326 de ce numéro). Deux autres familles de protéines de masse moléculaire voisine, également capables de lier et d'hydrolyser le GTP, présentent des caractéristiques relativement différentes des protéines des familles ras/rho/rab et leur sont reliées de façon beaucoup plus distante :

- la protéine ran/TC4, localisée dans le noyau des cellules, pourrait être impliquée dans les mécanismes de condensation de la chromatine [6] ;

- les protéines ARF, constituants majeurs du manteau des vésicules bourgeonnant de l'appareil de Golgi, joueraient un rôle important dans la formation et l'adressage des vésicules de transport [5] (article de Bruno Goud, p. 326).

Les petites protéines $\mathrm{G}$ des familles ras/rho/rab, bien que ne présentant pas de séquence hydrophobe, sont associées dans la cellule à des compartiments membranaires. Elles sont synthétisées sous la forme de précurseurs cytosoliques qui sont modifiés de façon post-traductionnelle, modifications qui sont responsables de l'attachement des protéines mûres aux membranes $[7,8]$. Les petites protéines $G$ des familles ras et rho, ainsi que certains membres de la famille $r a b$ possèdent à leur extrémité C-terminale une séquence de type $\mathrm{CAAX}$, où $\mathrm{C}$ représente une cystéine, $\mathrm{A}$ un résidu généralement aliphati- 
que et $\mathrm{X}$ un acide aminé quelconque. Dans un premier temps, la cystéine du motif CAAX est modifiée par l'adjonction d'un groupement isoprényle : le farnésyl à 15 atomes de carbone dans le cas des protéines ras et rap2 ou le géranylgéranyl à 20 atomes de carbone pour la plupart des autres protéines. Les trois derniers acides aminés (AAX) sont alors clivés, et la cystéine, à présent Cterminale, est carboxyméthylée. Toutefois, ces modifications ne semblent pas, à elles seules, suffisantes pour assurer l'attachement membranaire stable des petites protéines G. Certaines possèdent un ou deux autres résidus cystéine au voisinage de la séquence CAAX qui sont modifiés par palmitoylation ; d'autres présentent dans cette région une série d'acides aminés basiques qui pourraient interagir avec les têtes polaires des phospholipides. Dans le cas des protéines ras, deux signaux, à savoir isoprénylation et palmitoylation ou présence d'un motif polybasique, sont nécessaires à leur association avec la membrane plasmique. En revanche, la plupart des protéines de la famille rab possèdent à leur extrémité Cterminale une séquence de type CC ou CXC ; elles sont également modifiées par géranylgéranylation et il semble que certaines séquences de la région C-terminale de la molécule soient impliquées dans la localisation subcellulaire et l'association de ces protéines aux membranes [9].

Les protéines $G$ peuvent exister sous deux états conformationnellement différents, qui constituent respectivement les formes inactive et active de ces protéines selon qu'elles sont liées au GDP ou au GTP dans la cellule. Alors que la concentration intracellulaire en GTP est supérieure à celle en GDP d'environ un ordre de grandeur, les protéines ras sont normalement liées au GDP in vivo. Cela est dû à l'activité de protéines appelées GAP (GTPase activating protein) capables de stimuler plus de 100 fois l'activité GTPase intrinsèque des protéines ras [10]. Deux protéines possédant une telle activité ont été isolées : la p120-GAP, protéine cytosolique exprimée dans toutes les cellules, et le produit du gène de susceptibilité à la neurofibromatose de type $1, \mathrm{NF}-1$. La p120-GAP porte des domaines $m / s n^{\circ} 4$, vol. 8, auril 92
$\mathrm{SH} 2$ et $\mathrm{SH} 3$ caractéristiques des protéines susceptibles d'interagir respectivement avec des polypeptides comportant des tyrosines phosphorylées et des éléments du cytosquelette. Elle forme des complexes multiprotéiques avec des récepteurs de facteurs de croissance activés par leur ligand (récepteurs du PDGF ou du CSF-1), ainsi qu'avec des protéines impliquées dans le contrôle de la prolifération cellulaire telles que la phospholipase C- $\gamma$, la phosphatidylinositol-3 kinase, la $p p 60^{\text {src }}$ et la protéine kinase Raf-1 ; ces interactions pourraient ainsi constituer le lien biochimique entre les protéines ras et la transduction des signaux mitogènes des facteurs de croissance $[11,12]$. Le rôle physiologique des protéines p120-GAP et NF-1 n'est pas encore élucidé. Stimulant l'activité GTPase des protéines ras, elles se comportent comme un régulateur négatif qui les fait passer de leur état actif lié au GTP, à l'état inactif lié au GDP. D'autres arguments expérimentaux, fondés notamment sur les caractéristiques de l'interaction ras-GAP, et la nécessité de cette interaction pour certains effets des protéines ras, font au contraire penser que les protéines p120-GAP et NF-1 pourraient constituer les effecteurs responsables des effets biologiques des protéines ras $\left(\mathrm{m} / \mathrm{s} n^{\circ} 4\right.$, vol. 8, p. 388). L'action de ces deux protéines est restreinte aux protéines ras, et des protéines portant une activité GAP spécifique vis-à-vis d'autres petites protéines $G$ ont été mises en évidence. Il est remarquable que les protéines GAP propres aux protéines de la famille rho (rho, rac et $\mathrm{G} 25 \mathrm{~K} / \mathrm{CDC} 42 \mathrm{Hs}$ ) constituent elles-mêmes une famille de protéines qui comportent des homologies de séquence avec le produit du gène $b c r$, point de cassure dans la translocation réciproque formant le chromosome Philadelphie caractéristique de certaines leucémies myéloïdes chroniques et leucémies lymphoïdes aiguës [13].

L'activation des protéines $\mathrm{G}$ consiste en leur passage de la forme inactive liée au GDP à la forme active liée au GTP. Ce processus est dépendant de l'action d'un facteur qui stimule la dissociation du GDP (appelé GDS pour GDP dissociation stimulator) et accélère ainsi l'échange GDP. GTP [2]. De telles protéines, agissant chacune sur une ou un groupe restreint de petites protéines $G$, ont été mises en évidence. Elles interagissent généralement avec la région C-terminale des petites protéines $G$ après leur modification par isoprénylation. Il est à noter que la GDS spécifique de la protéine $\mathrm{G} 25 \mathrm{~K} / \mathrm{CDC} 42 \mathrm{Hs}$ est le produit du proto-oncogène $d b l$. Un autre type de protéine appelé GDI (GDP dissociation inhibitor) est capable de ralentir la dissociation du GDP de certaines petites protéines G. Ces GDI, décrites pour les protéines rho $B$ et rab3A, interagissent également avec l'extrémité C-terminale isoprénylée de ces protéines. Outre leur influence sur la vitesse de dissociation du GDP, les GDI sont capables, en se liant aux petites protéines $G$, de rompre leur attachement membranaire ; un tel phénomène joue probablement un rôle important dans les processus par lesquels les petites protéines $\mathrm{G}$ contrôlent certaines étapes du transit intracellulaire.

Les mécanismes par lesquels les petites protéines $G$ interviennent dans le contrôle de processus aussi importants pour la cellule que la prolifération, l'état du cytosquelette, le transit intracellulaire et la condensation de la chromatine, sont encore mal compris. Toutefois, de nombreuses avancées concernant la localisation subcellulaire et le mode d'interaction des petites protéines $G$ avec les membranes, ainsi que la mise en évidence et l'identification des protéines interagissant spécifiquement avec chaque petite protéine G (GAP, GDI, GDS), ont été récemment accomplies. Cette moisson d'informations nouvelles devrait bientôt nous permettre de comprendre, au niveau moléculaire, le mode d'action des petites protéines $G$ ainsi que leur rôle régulateur dans un nombre croissant de processus cellulaires importants

\section{TIRÉS A PART}

J. de Gunzburg 